\title{
Pulmonary function, inflammation, exercise capacity and quality of life in cystic fibrosis
}

\author{
J. Bradley*,\#, O. McAlister*,\#, S. Elborn*
}

\begin{abstract}
Pulmonary function, inflammation, exercise capacity and quality of life in cystic fibrosis. J. Bradley, O. McAlister, S. Elborn. (C)ERS Journals Ltd 2001.

ABSTRACT: The aim of the study was to determine the extent to which treatment induced changes in exercise capacity and quality of life $(\mathrm{QoL})$ are related to spirometric measures of lung function and other measures of disease impairment.

Twenty patients admitted to hospital with an exacerbation of pulmonary disease were recruited. Measures of disease impairment, disability and QoL were obtained at the beginning and end of an intravenous course of antibiotic therapy.

Intravenous antibiotic treatment resulted in a significant improvement in all measures of disease impairment, disability and handicap. The only significant predictor of treatment induced change in exercise capacity was $C$-reactive protein (CRP) and this explained $28 \%$ of the variance in change in exercise capacity. In the case of QoL, two predictors (change in exercise capacity and sputum output) contributed significantly to the change in QoL and collectively explained $54 \%$ of the variance in QoL.

Lung function provides a limited index of treatment outcome. Exercise capacity and quality of life assessment have the potential to make a significant contribution to the decision making process regarding treatment choices in cystic fibrosis and should be measured directly if a comprehensive evaluation of the effect of treatment is required.

Eur Respir J 2001; 17: 712-715.
\end{abstract}

Outcome measures are essential to monitor the effectiveness of current clinical practice and to determine the efficacy of new treatment strategies. The primary outcome measures used in cystic fibrosis (CF) are reflective of disease impairment, rather than the disability and handicap associated with the disease and it's treatment. This practice is based on the assumption that measures of disease impairment, particularly spirometric measures of lung function, relate well to the impact of a disease and its treatment on a patients life. Recent evidence has shown that this is not always the case in patients with $\mathrm{CF}$, or patients with other chronic lung diseases. The relationship between disease impairment (spirometry), disability (exercise capacity) and handicap (quality of life) has been shown to be inconsistent and frequently poor [1-6]. Interventions have also been shown to improve exercise capacity and quality of life $(\mathrm{QoL})$, independent of any changes in lung function $[7,8]$. These studies highlight that changes in disability and handicap may be influenced by mechanisms other than changes in spirometric measures of lung function.

The aim of this study was to determine the extent to which treatment induced changes in exercise capacity and QoL are accounted for by spirometric measures of lung function and other measures of disease impairment.
*Adult Cystic Fibrosis Unit, Belfast City Hospital and ${ }^{\#}$ University of Ulster at Jordanstown, Northern Ireland.

Correspondence: S. Elborn, Adult Cystic Fibrosis Unit, Belfast City Hospital, Lisburn Rd, Co. Antrim, Northern Ireland.

Fax: 441232263546

Keywords: Cystic fibrosis exercise capacity inflammation

pulmonary function quality of life

Received: February 72000

Accepted after revision September 13 2000

\section{Materials and methods}

\section{Study subjects}

Twenty patients admitted to hospital with an exacerbation of pulmonary disease were recruited. An acute exacerbation was defined as the presence of $\geqslant 4$ / 12 signs and symptoms of a respiratory exacerbation. These were: change in sputum production; new or increased haemoptysis; increased cough; increased dyspnoea; malaise; fatigue or lethargy; fever; anorexia or weight loss; sinus pain or tenderness; changes in sinus discharge; loss of appetite; $>10 \%$ (of best over previous 6 months) deterioration in lung function [9]. The decision to treat was made solely on the basis of the above clinical data. Inpatient treatment consisted of 14 days of parenteral antibiotic administration (guided by in vitro sensitivity of cultured organisms), intensified airway clearance, bronchodilator administration and nutritional support. No patient was prescribed oral steroids during the course of this experiment. The study was approved by the hospital ethical committee and informed consent was obtained from all patients.

\section{Study design}

Measures of disease impairment (forced expiratory volume in one second (FEV1), arterial oxygen 
saturation $\left(\mathrm{Sa}_{\mathrm{a}} \mathrm{O}_{2}\right)$, C-reactive protein (CRP), 24-h sputum weight and body weight), disability (modified shuttle test (MST) performance) and quality of life (Chronic Respiratory Disease Questionnaire (CRDQ)) were obtained at the beginning and end of an i.v. course of antibiotic therapy.

\section{Methods}

The measures of disease impairment recorded were those that reflect current clinical practice and included spirometric measures of lung function (Vitalograph Alpha, Vitalograph Ltd., Buckingham, UK), $S_{\mathrm{a}}, \mathrm{O}_{2}$, breathing room air (Ohmeda 3775 pulse oximeter Hatfield, UK), C- reactive protein (rate nephelometry), 24-h sputum weight (precision balance) and body weight.

To calculate 24-h sputum weight patients were given a number of plastic containers and asked to collect all sputum expectorated during a 24-h period following the beginning of $i$. v. antibiotic treatment and prior to the end of treatment. The MST and the CRDQ were used to measure disability and handicap, respectively.

All tests were administered at approximately the same time of day, at the beginning and at the end of $i$.v. antibiotic therapy. Patients were previously familiarized with all tests prior to entry into the study. The CRDQ administration and blood sampling for estimation of CRP were always carried out before exercise testing.

\section{Analysis}

Spearmans $\rho$ was used in the correlational analysis to investigate the relationship between variables of interest. The standardized response mean (SRM) was used to assess responsiveness of each of the outcome measures [10]. The SRM is the ratio of change in mean scores over time to the standard deviation (SD) of change ((mean scores at end of i.v. antibiotics (T2)mean scores at beginning of $i . v$. antibiotics) (T1)/SD of the change). The SRMs for each of the outcomes can be interpreted according to the guidelines outlined by MEENAN et al. [10]. An SRM of 0.2 is small, 0.5 indicates moderate sensitivity and an SRM of $\geqslant 0.8$ is considered highly responsive.

In order to determine the percentage of variance explained in QoL and exercise capacity by the other explanatory variables, least median regression was used as the basis for a reweighted least squares solution [11]. The first least squares regression model was set up with change in MST distance as the response variable and the other measures as the explanatory variables. A second similar model was set up with the only difference being that change in QoL was the response variable.

\section{Results}

Two out of 20 patients recruited did not complete the study (one patient refused to carry out the MST at discharge, the other patient developed acute cepacia syndrome and subsequently died) and therefore were not included in the data analysis.

Table 1 summarizes the physical characteristics, physiological data, data for exercise performance and QoL at beginning and end of $i . v$. antibiotic treatment. Within group analysis showed that there were significant improvements $(\mathrm{p}<0.05)$ in disease impairment (lung function, CRP, $\mathrm{Sa}_{\mathrm{a}} \mathrm{O}_{2}$, body weight and 24-h sputum weight), disability (exercise capacity) and handicap (QoL) following treatment.

A number of pathogens were cultured from their admission sputum (Burkholderia cepacia, $\mathrm{n}=6$; Pseudomonas aeruginosa, $\mathrm{n}=6$; Haemophilus influenza, $\mathrm{n}=6$; Staphylococcus aureus, n=6; Aspergillis fumugatus, n=1; Streptococcus pneumoniae, $\mathrm{n}=1$; Stenotrophomonas maltophilia, $\mathrm{n}=1$ ). Aminoglycoside antibiotics were administered i.v. in 15 exacerbations (gentamicin $(n=2)$, tobramycin $(n=13)$. The aminoglycoside antibiotics were administered in combination with ceftazidime $(n=6)$, aztreonam $(n=2)$, tazobactam $(n=3)$, or merepenum $(n=4)$. In three exacerbations monotherapy was used (cefuroxime $(n=1)$ and cefotaxime $(n=2)$. In four patients additional oral antibiotics were also used (flucloxacillin, ciprofloxacin and co-trimoxazole).

There was a moderate and significant relationship between exercise performance and FEV1 at T1 $(\rho=0.70$, $\mathrm{p}<0.01)$ and T2 $(\rho=0.71, p<0.01)$ but the relationship between change in FEV1 and change in exercise performance was poor $(\rho=-0.37, p=0.12)$. The relationship between lung function and QoL was poor and nonsignificant at T1, T2 and in terms of change. There was a moderate correlation between CRP and FEV1 at T1 $(\rho=-0.51, p<0.03)$ and T2 $(\rho=-0.53, p<0.02)$ but, a poor correlation between change in FEV1 and change in CRP $(\rho=0.29, p=0.25)$. There was a weak negative

Table 1. - Physical characteristics, physiological exercise performance and QoL data at the beginning and end of i.v. antibiotic treatment $(n=18)$

\begin{tabular}{lcccc}
\hline & $\begin{array}{c}\text { Beginning of } \\
\text { treatment } \\
\text { mean } \pm \text { SD }\end{array}$ & $\begin{array}{c}\text { End of } \\
\text { treatment } \\
\text { mean } \pm \text { SD }\end{array}$ & $\begin{array}{c}\text { Mean } \\
\text { Diff }\end{array}$ & p-value \\
& $23 \pm 5$ & & & \\
Age yrs & $57 \pm 11$ & $58 \pm 10$ & 1 & 0.001 \\
Weight kg & $1.8 \pm 0.8$ & $2.3 \pm 1.1$ & $\begin{array}{c}(0.2-2) \\
0.5\end{array}$ & $<0.01$ \\
FEV1 L·min ${ }^{-1}$ & $49 \pm 17$ & $60 \pm 25$ & $\begin{array}{c}(0.2-0.7) \\
11\end{array}$ & $<0.01$ \\
FEV1 \% pred & $26 \pm 19$ & $10 \pm 14$ & $\begin{array}{c}(6-16) \\
-16\end{array}$ & $<0.01$ \\
Weight sputum g & $16 \pm 18$ & $2 \pm 2$ & $\begin{array}{c}(-9-22) \\
(-5-23)\end{array}$ & $<0.01$ \\
CRP & $94 \pm 3$ & $95 \pm 3$ & $\begin{array}{c}1 \\
(0.5-2)\end{array}$ & $<0.01$ \\
Sa,O $\%$ & $860 \pm 366$ & $1024 \pm 333$ & 164 & $<0.01$ \\
MST distance m & & & $(92-236)$ & \\
& & & & \\
QoL & $61 \pm 15$ & $81 \pm 13$ & $(12-28)$ & $<0.01$ \\
& & & & \\
\hline
\end{tabular}

95\% CI: 95\% confidence interval; FEV1: forced expiratory volume in one second; CRP: C-reactive protein; $\mathrm{Sa}_{\mathrm{a}, \mathrm{O}_{2}}$ : arterial oxygen saturation; MST: modified shuttle test; QoL: quality of life. 
Table 2. - The final reweighted squares solution with modified shuttle test distance as the response variable and the other variables as the explanatory variables

\begin{tabular}{lcccc}
\hline Predictor & $\begin{array}{c}\text { Unst. } \\
\text { coefficient }\end{array}$ & $\begin{array}{c}\text { St. } \\
\text { coefficient }\end{array}$ & t-value & p-value \\
\hline Intercept & 108.08 & & 2.66 & 0.02 \\
Change in CRP & -0.45 & -0.53 & -2.42 & 0.03 \\
$\mathrm{R}^{2}$ & & & & 0.28 \\
\hline
\end{tabular}

Unst.: Unstandardized; St.: standardized.

correlation between CRP and MST at T1 $(\rho=-0.26$, $p=0.29)$ and at T2 $(\rho=-0.34, p=0.17)$ but there was a stronger negative correlation between the change in CRP and MST $(\rho=-0.50, p=0.04)$. The relationship between CRP and QoL was also poor (T1: $\rho=0.26$, $p=0.31$; T2: $\rho=-0.02, p=0.95$; change: $\rho=-0.03, p=0.93$ ).

All of the outcome measures were either moderately (CRP: SRM=0.78) or highly responsive $(\mathrm{FEV} 1 \%$ predicted: SRM=1.03; MST distance: $S R M=1.12$; QoL: $\left.\mathrm{SRM}=1.28 ; \mathrm{Sa}_{2} \mathrm{O}_{2}: \mathrm{SRM}=0.86\right)$ to $i . v$. antibiotic treatment induced changes in clinical status.

The results of the regression analysis are shown in tables 2 and 3 . In the case of exercise capacity the only significant predictor of treatment induced change in exercise capacity was CRP. The multiple coefficient of determination $\left(\mathrm{R}^{2}\right)$ implies the amount of variation in the response variable that can be explained by the explanatory variables. Change in CRP explained 28\% of the variance in change in MST distance. If the other variables had been forced into this model they would have explained only a small and insignificant proportion of the variance in MST distance.

In the case of QoL, two predictors (change in MST and sputum output) contributed significantly to the change in QoL and collectively explained 54\% (change in MST explained 27\%; change in sputum output explained an additional $27 \%$ ) of the variance in change in QoL. If the other variables had been forced into this model they would have explained only a small and insignificant proportion of the variance in MST distance.

\section{Discussion}

According to the World Health Organization (WHO) the impact of any condition can be classified using the international classification of impairment, disability and handicap [12]. The assessment tools that are

Table 3. - The final reweighted squares solution with quality of life as the response variable and the other variables as the explanatory variables

\begin{tabular}{lcccc}
\hline Predictor & $\begin{array}{c}\text { Unst. } \\
\text { coefficient }\end{array}$ & $\begin{array}{c}\text { St. } \\
\text { coefficient }\end{array}$ & t-value & p-value \\
\hline Intercept & 0.55 & & 0.12 & 0.90 \\
Change in distance & 0.04 & 0.55 & 2.80 & 0.01 \\
$\begin{array}{l}\text { Change in weight } \\
\text { sputum }\end{array}$ & -0.47 & -0.52 & -2.66 & 0.02 \\
$\mathrm{R}^{2}$ & & & & 0.54 \\
\hline
\end{tabular}

Unst.: Unstandardized; St.: standardized. potentially available for the clinical evaluation of patients with $\mathrm{CF}$ can be subdivided into the assessment of these three areas. The most commonly used measurement tools in $\mathrm{CF}$ focus on the assessment of impairment, for example spirometric assessment of lung function. This study investigated whether there is justification for the use of spirometry as the sole index of the impact of treatments on CF disease. The model of an i.v. antibiotic treatment was used to investigate whether the relationship between spirometry and measures of disability and handicap was such that measures of disability (exercise capacity) and handicap (QoL) could be predicted reliably from spirometry.

The results show that i.v antibiotic treatment resulted in a significant improvement in all measures of disease impairment, disability and handicap. As the SRMs are standardized scores, the SRM for each of the measures of impairment, disability and handicap can be directly compared. These values showed that all the measurements were sensitive to $i$. $v$ antibiotic treatment and no one measurement was clearly best. The regression analysis showed that lung function explained only a small and nonsignificant proportion of $i . v$ treatment induced changes in exercise capacity and QoL. This is consistent with the findings of the correlational analysis between change in spirometric measures of lung function and change in exercise performance and QoL. Table 2 shows that only CRP contributed significantly to treatment induced change in disability and only change in MST and sputum output contributed significantly to the change in QoL. A large percentage of the variance in disability and QoL was not addressed by outcome measures frequently used to assess the efficacy of new treatments in $\mathrm{CF}$ and to compare the effectiveness of new versus existing treatment strategies.

These findings are important because they show that no one factor exclusively explained the impact of $i . v$ antibiotic treatment on an acute exacerbation of pulmonary disease. Lung function provides a limited index of the outcome of treatment. Other measures of disease impairment contribute to the percentage of explained variance in disability and handicap, but as a considerable amount of variance is not addressed by commonly used measures, direct measurement of exercise capacity and QoL are necessary to comprehensively assess the net effect of $i$. $v$ antibiotic treatment in CF. A plethora of instruments are available to measure QoL and exercise performance. The tests used in this study have been used previously in CF and information is available regarding the psychometric properties of these tests in CF [13-15].

The reasons for these findings can be attributed, at least in part, to the complexity of the pathophysiology of lung disease. Patients with $\mathrm{CF}$ range from being asymptomatic with few signs of ill health, to having a chronic cough, productive of large amounts of sputum, a wheeze, dyspnoea and limited exercise tolerance. Some of this variability may be attributable to specific gene mutations, but environmental factors and treatment strategies also have a large role to play $[16,17]$. In addition, the physical, social and emotional effects of these clinical manifestations are specific to the individual. For example, the disability incurred by a 
patient with moderate disease impairment will be less in the patient who leads a sedentary lifestyle than one who is very active [18]. Similarly the handicap imposed by the disease also depends on the individual; their own circumstances, perceptions, fears, aspirations and more importantly their own disease. Handicap in $\mathrm{CF}$ may be much greater in a patient who leads a very active lifestyle and has family, work and social commitments, than in one who has a very sedentary lifestyle. Therefore, it is not surprising that no single measure of disease impairment such as lung function can satisfactorily summarize the various disturbances caused by the signs and symptoms of CF.

Exercise capacity and sputum production had a significant impact on a patients perception of their QoL. The systemic inflammatory response to infection, as indexed by CRP, may have a direct impact on treatment induced change in exercise capacity in CF. It may also impact the outcome of treatment in $\mathrm{CF}$ via other pathways such as increased sputum production. Regardless of the exact mechanisms involved these findings highlight the importance of measuring, directly, the outcome of treatment which should focus not only on strategies that target lung disease directly, but also on interventions that improve sputum production, modulate the inflammatory response to infection and improve exercise tolerance and QoL.

Published research in other respiratory diseases have also shown that the relationship between spirometric measures of lung function, disability and handicap are such that percentage of shared variance between change in exercise capacity and change in lung function and between change in handicap and change in lung function are small [4-6]. Directly targeting the systemic response to infection may also be important in maximizing treatment efficacy in general respiratory diseases.

The authors are not advocating that measures of disability or handicap replace spirometry, nor are they suggesting that spirometry does not contribute to the measurement of the outcome of $i . v$ antibiotic treatment. Rather, this work highlights that exercise and QoL data provides information regarding the outcome of treatment that is not explained by a change in spirometry. These findings have important implications for design of clinical trials to assess the efficacy of new treatments, for the clinical evaluation of treatments and for decisions regarding treatment choices in $\mathrm{CF}$.

Exercise capacity and quality of life have the potential to make a significant contribution to the decision making process regarding treatment choices in cystic fibrosis. Any evaluation of a new treatment in cystic fibrosis must justify not using measures of disability and handicap, in addition to measures of disease impairment. Spirometry cannot be used as a surrogate measure of changes in disability or handicap. These should be measured directly if a comprehensive evaluation of the effect of treatment is required.

\section{References}

1. Webb AK, Dodd ME. Exercise and cystic fibrosis. $J R$ Soc Med 1995; 88: 30-36.

2. Congleton J, Hodson ME, Duncan-Skingle F. Do NHP scores change over time in cystic fibrosis. Respir Med 1998; 92: 268-272.

3. Glyzewski D, Marriotto MJ, Bartholomew K. Measurement of the quality of wellbeing in a child and adolescent cystic fibrosis population. Med Care 1994; 32: 965-972.

4. Maille AR, Kaptein AP, de Haes JCJM, Everaerd WTAM. Assessing QoL in chronic non specific lung disease. A review of empirical studies published between 1984 and 1994. Qual of Life Res 1996; 5: 287-301.

5. Jones PW, Quirk FN, Baveystock CM. The St George's Respiratory Questionnaire. Respir Med 1991; 83 (Suppl. B): 25-31.

6. Jones PW, Quirk FN, Baveystock CM. A self complete measure of health status for chronic airflow limitation - The St Georges Respiratory questionnaire. Am Rev Respir Dis 1992; 145: 1321-1327.

7. Reisman J, Schneiderman J, Walker Corey M, et al. The role of an organized exercise program in cystic fibrosis. A three year study. Pediatr Pulmonol 1995; A252.

8. de Jong W, Kaptein AA, VanderSchans CP, et al. Quality of life in patients with cystic fibrosis. Pediatr Pulmonol 1997; 23: 95-100.

9. Ramsey BW, Pepe M, Williams WJ. Pulmonary exacerbation (PE): How do we define it? Pediatr Pulmonol 1994; (Suppl. 9): 77-78.

10. Meenan R, Karis L, Anthony J, Wallis B. The clinical and health status of patients with recent onset of rheumatoid arthritis. Arthritis Rheumatol 1991; 347: $61-65$.

11. Rousseeuw PJ, Leroy AM. Robust regression and outlier detection. New York, Wiley, 1987; 75-125.

12. WHO International classification of impairment, disability and handicap. WHO 1980, Geneva.

13. Bradley JM, Dempster MT, Wallace ES, Elborn JS. The adaptations of a quality of life questionnaire for routine use in clinical practice: the chronic respiratory disease questionnaire in cystic fibrosis. Qual of Life Res 1999; 6: 65-71.

14. Bradley JM, Howard JL, Wallace ES, Elborn JS. Validity of a modified shuttle test in adult cystic fibrosis. Thorax 1999; 49: 437-439.

15. Bradley JM, Howard JL, Wallace ES, Elborn JS. Reliability, repeatability and sensitivity of the modified shuttle test in adult cystic fibrosis. Chest 2000; 117: 1666-1671.

16. Kerem E, Reisman J, Corey $\mathrm{M}$, et al. Prediction of mortality in patients with CF. N Engl J Med 1992; 326: $1187-1191$.

17. Tullis DE, Guyatt GH. Quality of Life in Cystic Fibrosis. PharmacoEconomics 1995; 8: 22-33.

18. Morgan MDL. Practical Pulmonary Rehabilitation: An Introduction. In: Morgan MDL, Singh SJ, eds. Practical Pulmonary Rehabilitation. London, Chapman and Hall, 1997; 1-18. 